\title{
Du bon usage des motifs légendaires. A propos du conte-type 713
}

\author{
Nicole Belmont \\ Laboratoire d'Anthropologie Sociale, Paris \\ nicole.belmont@college-de-france.fr
}

RÉSUMÉ

Le conte $A T U$ 7I3, La mère qui ne m'a pas porté, mais m'a nourri, introduit par Paul Delarue dans la typologie internationale, comporte un petit nombre de versions, toutes françaises (Nivernais et sud-ouest) à l'exception d'un récit provenant de l'île de Majorque. Dans cet article nous faisons l'hypothèse de la création relativement récente de ce conte dans la région du Nivernais et du Morvan qui, au XIXe siècle, fournissait un nombre important de nourrices. Cette activité contraignait les femmes à un exil temporaire, mais leur permettait d'apporter une aisance financière à leur retour au pays: le conte sublime cette servitude en utilisant le motif de l'abondance liée à la présence de l’héroïne. Le récit, introduit dans le sud-ouest de la France où n'existaient pas les mêmes contraintes économiques, a pu s'y implanter, de façon précaire cependant, en introduisant un motif légendaire, lequel permet paradoxalement de maintenir son statut de conte merveilleux.

\section{MOTS CLÉS}

Conte merveilleux ; motif hagiographique ; Nivernais (France) ; maternité procréatrice; maternité nourricière

ABSTRACT

The folktale ATU 7I3, The Mother Who Did Not Bear Me but Nourished Me, introduced to the international catalogue by Paul Delarue, includes a small number of versions, all French (Nivernais and South-West) apart from one story from the island of Mallorca. In this paper we put forward the hypothesis that this tale was created relatively recently 
in the region of Nivernais and Morvan which, in the nineteenth century, was home to a large number of nurses. This activity forced women into temporary exile, but allowed them to generate a certain level of financial comfort for their return to their place of origin. The tale sublimates the heroine's servitude with the abundance for which she has left her home to work. The story was subsequently introduced to the south-west of France, which did not have the same economic constraints, and it thus evolved to include, albeit precariously, a legendary motif which paradoxically allows it to maintain its status as a tale of magic.

\section{Keywords}

Tale of magic; hagiographic motive; Nivernais (France); procreative motherhood; foster motherhood

\section{RESUM}

La rondalla $A T U$ 7I3, El pa convertit en flors, introduïda per Paul Delarue en la tipologia internacional, té un nombre reduït de versions, totes franceses (Nivernais i sud-oest), amb l'excepció d'una història de l'illa de Mallorca. En aquest article fem la hipòtesi de la creació relativament recent d'aquesta rondalla a la regió de Nivernais i de Morvan, que al segle XIX va acollir un gran nombre d'infermers. Aquesta activitat va obligar les dones a l'exili temporal, però els va permetre aportar una facilitat financera al seu retorn al país: la història sublima aquesta servitud amb la raó de l'abundància vinculada a la presència de l'heroïna. La història, introduïda al sud-oest de França, on no existien les mateixes restriccions econòmiques, va ser capaç d'introduir, encara que precàriament, un motiu llegendari que paradoxalment permet mantenir la seva condició de rondalla meravellosa.

Paraules clau

Rondalla meravellosa; motiu hagiogràfic; Nivernais (França); maternitat procreativa; maternitat adoptiva 
onte intrigant malgré le peu de versions qu'on en connaît, La mère qui ne m'a pas porté, mais m'a nourri (ATU 7I3) pourrait nous faire entrevoir, en dépit de son caractère précaire, certains des mécanismes de la formation et de l'élaboration des contes merveilleux. On fera l'hypothèse que sa survie hors de son milieu d'origine présumé a été assurée par le recours à un motif légendaire, motif hagiographique, sans lequel, de façon paradoxale, il n'aurait pu maintenir sa structure de conte merveilleux. ${ }^{\mathrm{I}}$

Le faire-part de naissance de ce conte-type a paru dans la revue Fabula en I959. Signé par Paul Delarue, cet article était malheureusement posthume (Delarue I959). ${ }^{2}$ Il y notifiait l'insertion dans son catalogue des contes français d'un récit non répertorié dans la typologie internationale. "Je propose de lui attribuer, au voisinage des contes-types de la série "the banished wife or maiden", le n encore disponible 7I3 ». Il en dénombrait onze versions recueillies dans le Nivernais par Achille Millien et trois autres provenant du sud-ouest de la France; à ces dernières, il faut maintenant en ajouter deux de plus provenant respectivement de l'Hérault et des Landes (Bru et Bonnemason 20I7: 666-669). Conte exclusivement français, déclarait P. Delarue après enquête auprès de chercheurs étrangers. Cependant Josep A. Grimalt en a découvert une version dans le corpus recueilli par Antoni M. Alcover à Majorque, mais non publié par lui dans son recueil : précieux témoignage hors du domaine français (Grimalt I986). En dépit de ce petit nombre, Stith Thompson et Hans-Jörg Uther à sa suite ont entériné l'insertion de ce récit dans la typologie internationale. ${ }^{3}$

La découverte de P. Delarue provient à l'origine de sa connaissance approfondie du fonds Achille Millien (I838-I927), la plus grande collecte de contes et de chansons jamais réalisée en France (Nivernais et Morvan), qu'il classera entièrement et publiera en partie. Ces onze versions nivernaises ${ }^{4}$ sont lacunaires, voire elliptiques pour certaines, au point que P. Delarue avait composé une version synthétique (I956). ${ }^{5}$ Il y puisera un résumé pour son article de I959.

Un roi qui a une fille d'un premier lit, Brigitte, se remarie à une femme qui a aussi une fille.

Brigitte est très bonne et donne beaucoup aux pauvres. Un jour, comme elle leur porte du pain dans son tablier, sa belle-mère survient pour la gronder:

— Que portes-tu dans ton tablier?

- Des fleurs.

Elle regarde: le pain s'était changé en fleurs.

I Ce texte quelque peu modifié a été repris dans Nicole Belmont (20I7 : 57-79) chapitre 3, "La "fabrication" d'un conte merveilleux : "Brigitte, la mère qui ne m'a pas fait mais m'a nourri"».

2 Il fut transmis à Kurt Ranke par Marie-Louise Tenèze.

3 La révision de Uther a inclus le T $7 \mathrm{I}^{*}$ Meat Stolen for Poor Turns to Roses dans notre conte qui, en effet, l'utilise parfois, mais à titre d'épisode non contraignant.

4 Sept ont été recueillies dans la vallée de la Nièvre, deux dans le nord de la province, deux à l'est, « dont une aux confins de la Bourgogne, à Glux » (Delarue I959: 254).

5 Reprise par M.-L. Tenèze pour le catalogue des contes français. 
La fille de la marâtre se conduisait mal, et une nuit, elle accouche d'un enfant. La reine le porte dans le lit de Brigitte et va chercher le roi, qui, croyant sa fille coupable, la chasse avec le nouveau-né.

Brigitte arrive à un moulin, demande de la farine pour faire de la bouillie à l'enfant:

- Il y a sept ans que, faute de blé, notre moulin ne marche plus.

Brigitte cherche, trouve un grain de blé, le met dans le moulin ; celui-ci tourne et donne de la farine à pleins sacs.

Elle demande ensuite du lait:

— Nos vaches n'en ont plus.

- Allez les traire.

On y va : les vaches donnent du lait à pleins seaux. L'abondance règne désormais dans le pays.

En revanche, au pays du roi, depuis le départ de Brigitte, l'herbe ne pousse plus, les récoltes sont mauvaises, les bestiaux sont malades.

Le roi part à la recherche de sa fille, arrive près du moulin, voit un enfant qui pêche:

— Que fais-tu, mon enfant?

— Je pêche pour maman Brigitte qui m'a pas fait mais m'a nourri.

— Conduis-moi vers elle.

Le père retrouve sa fille, la ramène.

En route, l'enfant cueille trois pommes. Ils arrivent. Au pays du roi, les prés reverdissent, le bétail guérit.

On se met à table. À la fin du repas, l'enfant offre ses trois pommes:

- Une pour maman Brigitte qui m'a pas fait, mais m'a nourri... Une pour maman qui m'a fait mais m'a pas nourri... Une pour grand-père...

Le roi chasse la reine et sa fille (Delarue I959: 255).

Si, pour P. Delarue, le motif de la fille persécutée par sa marâtre, injustement accusée, puis bannie, assorti ou non du motif du pain changé en fleurs, est bien présent dans les contes féminins, le fait que l'héroïne soit chassée avec un enfant qui n'est pas le sien, mais qu'elle accepte d'élever (de nourrir en tout premier) « constitue un motif très nettement particularisé » (Delarue I959: 256). Les conteurs et conteuses qui sentaient aisément une affinité entre ce récit et La Fille aux mains coupées (T 706), ne se trompaient jamais quant à l'origine de l'enfant dont l'héroïne a la charge lors de son bannissement: dans ce cas celui d'une autre femme, dans l'autre ses propres enfants. Quelques versions du T 7I3 ajoutent cependant aux malheurs de l'héroïne l'ablation des mains, sans véritable motivation interne. Le second trait narratif original concerne le motif de la fertilité et de 
l'abondance liées à la présence de l'héroïne et la stérilité qui affecte le pays dont elle a été chassée. ${ }^{6}$

C'est le motif du pain changé en fleurs qui a orienté les recherches de P. Delarue vers l'hypothèse d'une origine légendaire, mais en portant tout particulièrement son attention sur le corpus concernant sainte Brigitte, dont l'héroïne porte le nom dans quatre des versions nivernaises. À la sainte irlandaise est attaché un riche corpus légendaire, qui témoigne de ses liens étroits avec l'abondance et la fertilité, en cela incontestable héritière de la divinité celtique Brigid. ${ }^{7}$ De nombreux récits racontent comment elle crée l'abondance. Ainsi, lors d'une assemblée, « on ne dispose que d'un mouton, de douze pains et d'un peu de lait pour une foule immense; mais Brigitte prie sur la nourriture qu'elle distribue, qui se trouve multipliée et suffit à rassasier tout le monde » (Delarue I959: 26I). Cependant l'initiateur du catalogue des contes français met en garde ses lecteurs:

on ne saurait assez répéter qu'un conte est un ensemble organique complexe qui peut combiner des éléments de provenance et d'âge divers dont aucun n'éclaire à lui seul la naissance toujours obscure du conte (Delarue I959: 257).

Le fonds légendaire concernant la sainte Brigitte irlandaise est constitué par un « conglomérat » d'épisodes divers, voire dispersés, recueillis principalement dans deux ouvrages, La Légende dorée et le Livre de Linsmore. Rien ne suggère la forme narrative « organique » qui est celle du conte merveilleux.

La découverte tardive de ce conte, la rareté de ses versions, concentrées en outre dans le domaine français à une exception près, le caractère lacunaire de certaines d'entre elles laissent penser qu'il est de création relativement récente. Son étude en est d'autant plus intéressante. Si nous n'avons pas l'ambition de répondre à la question de savoir comment naît un conte merveilleux, nous proposerons une hypothèse concernant la genèse de celui-ci, mais aussi de ce qu'on est tenté d'appeler sa précarité, même si la disparition de la transmission orale ne nous permet pas d'en apporter des preuves formelles.

La plus grande fréquence du récit dans la région du Nivernais et du Morvan témoigne tout d'abord de l'intensité de la collecte menée par Achille Millien dans le dernier quart du XIX ${ }^{\mathrm{e}}$ siècle. ${ }^{8}$ Elle met également en évidence l'absence du conte dans d'autres régions françaises, à l'exception des versions collectées dans le sudouest du pays. ${ }^{9}$ Le cadre de cet article ne permettra pas une analyse détaillée de

6 P. Delarue dit n'en connaître qu'un exemple, celui d'un conte kabyle « composite » recueilli par Frobenius, qui concerne un jeune homme banni et raconte une tout autre histoire. Mais le motif de la « gaste terre » est connu dans la littérature médiévale.

7 Sur la légende de la sainte irlandaise, on consultera l'article de Donatien Laurent (I982). Il existe une autre sainte Brigitte, d'origine suédoise, qui vécut au xive siècle.

8 Nous avons utilisé les transcriptions faites par Jacques Branchu que nous remercions vivement.

9 J'en dénombre quatre, ne tenant pas compte de la version Moulis ni de celle de Piniès, présentes dans le catalogue Delarue-Tenèze, mais qui relèvent clairement du T 706, La Fille aux mains coupées, tout en incluant le motif de l'abondance liée à la présence de l'héroïne. La proximité des deux contes-types est confirmée par G. Maugard dans ses commentaires à la première publication de son récit (1952: 27-28). Parlant également du T 706, il déclare: « ces contes nous ont été livrés la même nuit, très voisins, presque jumeaux - comme un deuxième aspect du cycle de Brigitte. La conteuse [sa grand-mère] hésitait entre les deux 
ces quinze versions, à laquelle on joindra celle que J. Grimalt a eu le bonheur de nous faire connaître. On tentera de souligner les traits susceptibles d'élucider la naissance tardive de ce conte. ${ }^{\text {Io }}$

Souvent lacunaires, on l'a dit, les onze versions nivernaises présentent cependant un grand intérêt. P. Delarue notait que quatre d'entre elles nomment

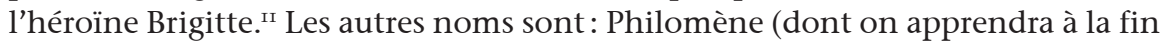
qu'elle est une sainte et qui, pour cette raison même, meurt à la fin du récit), Bélisaire, sainte Guite et Maman Guite pour deux autres. ${ }^{12}$ Trois versions ne nomment pas l'héroïne. L'absence du nom Brigitte n'entraîne nullement la disparition du motif de l'abondance engendrée par la présence de l'héroïne, signalant ainsi que le lien avec la sainte irlandaise n'est plus ressenti même s'il a existé. Un seul élément légendaire, le pain changé en fleurs, apparaît dans trois versions. Parmi celles-ci, il faut signaler en outre un souvenir évident de la légende de «Geneviève de Brabant » («Brizide»). Le père de l’héroïne, convaincu de sa culpabilité, la fait emmener avec l'enfant par deux valets chargés de la tuer. Ceux-ci l'épargnent, mais l'abandonnent dans la forêt. Elle appelle: «- Biche, biche, viens à toi / Je voi-t-au bois. Et la biche vint. Là Brizide vivait aux racines ». Lorsque son père veut la faire revenir: «Un des deux valets trouve [Brizide]: — Biche, décachez-vous! — Non, je suis trop couverte de poils ». Une fois rentrée chez son père, «Brizide ne voulait rien que des racines et de l'eau claire. On va lui en chercher, elle mange ». Souvenir de «Geneviève de Brabant » certes, mais réélaboré jusqu'à ensauvager l'héroïne, presque métamorphosée en animal. ${ }^{13}$ Les versions nivernaises comportent peu d'éléments d'origine légendaire inscrits dans leur trame, mais toutes incluent sans exception le motif de l'abondance et de la disette liées à la présence et à l'absence de l'héroïne, y compris les plus brèves qui ne comportent que cet épisode («Sainte Guite » et «Maman Guite »). Ainsi dans « Philomèle »:

Elle arrive dans un domaine. - Madame, si v'avins [vous aviez] donc la bonté de me donner un peu de lait pour la bouillie à mon enfant. — V'la sept ans, que nos vaches n'ont pas de lait. - Essayez. [La femme] est allée, ne pouvait pas les tarir. Elle revient. - Tenez, voilà du lait, mais point de farine. - Vous avez un moulin? - Non. Il n'a pas tourné depi sept ans. - Allez-y; vous en trouverez peut-être un peu moulu dans la corne de vot' maie. Elle y va, trouve la maie pleine de farine et le moulin moulait (Ms Millien, version 6).

\footnotetext{
saints [sic], car le départ est le même, et la thaumaturgie ne déparerait pas ce récit ». Dans ce cas, l'héroïne du T 706 se nomme Brigitte et celle du T 713 Germaine.

Io La version de Bladé, «La Petite demoiselle », qui perd rapidement son droit fil narratif, ne nous a été d'aucune utilité. Elle témoigne cependant de cette tentative de pénétration du récit dans le sud-ouest de la France.

II Brigitte pour deux d'entre elles; saint Brigire [sic] pour une autre, Brizide pour la dernière.

I2 Un excellent connaisseur du Morvan, Rémi Guillaumeau, nous a suggéré que ce nom de Guite pourrait être une autre forme de Guéte, autrement dit sainte Agathe, patronne des nourrices.

I3 La version lacunaire intitulée « Sainte Brigire » insère sans plus développer le nom de Golo, le traître légendaire dans « Geneviève de Brabant »: " [La belle-mère] la fait porter au bois. Golo. Elle retourne dans la forêt, y reste encore dix ans, s'en va dans un moulin, demande du lait pour faire la soupe à son enfant ».
} 
Deux des quatre versions du sud-ouest, à laquelle il faut joindre celle d'Alcover publiée par J. Grimalt, incluent le motif légendaire du pain changé en fleurs (Bladé et Maugard). Alors que celles d'Arnaudin, de Maugard, de Petit et d'Alcover en introduisent un autre, surprenant puisqu'il semble aller à l'encontre de la logique narrative du conte-type. Dans la première, "Virginette ", Berginète en gascon, il est raconté classiquement que la fille de la marâtre, qui « se tenait fort mal », accouche d'un enfant que sa mère porte aussitôt dans le lit de l'héroïne. Le matin, la «tante » appelle le père pour lui faire constater le fruit de la faute.

Son père se mit à l'appeler — Virginette ! Virginette! Réveille-toi... La fillette ne se réveillait jamais. — Et qu'as-tu donc fait, petite? — Qu'est-ce que j'ai donc fait, papa? dit-elle en ouvrant les yeux. — Oui, regarde ce que tu as, à côté de toi... Un enfant. - Un enfant, papa? Oui, tu ne sais peut-être pas d'où il te vient? - Non, papa, je n'en sais rien. Mais si le bon Dieu me l'a envoyé, il me donnera aussi de quoi le nourrir. La jeune fille se leva, s'agenouilla au pied du lit et se mit à prier Dieu. Aussitôt qu'elle eut fini sa prière, le lait monta dans sa poitrine et elle se mit à faire téter le petit enfant (Arnaudin I994, $\mathrm{n}^{\circ} \mathrm{xxx}$ ).

La version des Pyrénées (Maugard) raconte ainsi l'épisode.

A quelque temps de là, la fille de cette femme [la marâtre] mit au monde un bâtard. De qui était-il ? Je ne saurais le dire... C'était vers minuit. La marâtre porta le nouveau-né dans le lit, aux côtés de sainte Germaine. Au matin, elle découvrit le petit garçon. Le bébé avait faim et pleurait. - Pauvre ange, je ne sais d'où tu es sorti... Quoi qu'il en soit, je veux te donner mon sein... Seigneur, dit-elle faites que j'aie du lait afin de nourrir ce petit ange. Aussitôt il lui vint du lait (Maugard I955: I76).

Le même épisode se retrouve dans la version racontée par Germana Cabrol:

Et, un jour, il y en eut une, de ces filles [de la marâtre], qui eut un enfant, et dans la nuit elle va le porter près de Sainte Brigitte. Elle dit « Puisque Notre Seigneur m'a donné un petit, qu'il me donne du lait pour le nourrir ». Et elle eut du lait et le lendemain tous la réprimandèrent. Ils disaient « Regarde là comme elle est sainte, tiens. Elle a eu un enfant! » Enfin, on la réprimanda tellement qu'elle prit cet enfant dans son tablier et s'en va (Petit I974).

Dans la version majorquine, il s'agit d'un roi qui a eu une fille d'un premier mariage. Après la mort de son épouse, il se remarie avec une femme qui hait sa belle-fille car elle est jalouse de sa beauté. Elle vole un nouveau-né, le place dans le lit de la jeune fille et fait venir le roi pour constater sa culpabilité. La princesse prend l'enfant et dit: «— Si seulement j'avais des vêtements, je l'habillerais. Des vêtements apparaissent et elle habille l'enfant. - Si seulement mes seins avaient du lait, je le nourrirais. Du lait sort de sa poitrine et elle l'allaite ». J. Grimalt propose d'attribuer le $\mathrm{n}^{\circ}$ 6II.5.4: Child miraculously suckled by virgin à ce motif, à la suite de l'item du Motif-Index, T 6II.5: Child miraculously suckled by saint.. ${ }^{14}$

I4 Nous renvoyons à la passionnante étude de J. Grimalt, non sans signaler que le récit manuscrit d'Alcover porte deux titres: «N'Aineta, fia de Rey » (Annie, la fille du roi) et «Una fia de rey » (Une fille de roi). Sans oublier des informations elliptiques: un nom propre (Catalina Balaguer), un lieu (Estallencs) et la remarque « la dida de Dona Antonia » (la nourrice 
Ces versions n'omettent cependant pas le motif de la stérilité qui frappe le pays auquel elle parvient. La version majorquine relate bien que les habitants du pays où arrive l'héroïne, n'ayant plus de pain depuis sept ans, vivent d'herbe et couchent sur la paille, carence palliée par l'héroïne. Aussitôt après, est signalée la stérilité qui règne au royaume du père, provoquée par l'absence de pluie et que le peuple attribue à une faute du roi. Dans la version landaise, le motif est moins explicite. Il ne concerne pas tout un territoire, mais une maisonnée: la seule fontaine qui donne de l'eau est très éloignée. Virginette révèle l'existence de deux cruches pleines. L'hôtesse prépare alors du pain, dont l'héroïne a un grand désir, n'en ayant pas mangé depuis sept ans. ${ }^{15}$ Le petit morceau de pâte gonfle miraculeusement et donne un gros pain, une fois cuit. Dans le conte pyrénéen, L'héroïne, chassée par son père, arrive à un moulin dont les habitants n'ont plus rien à manger. Elle fait apparaître du petit salé et du pain : "Ils étaient bien pauvres auparavant, mais à partir de ce jour ils ne manquèrent plus de rien » (Maugard I955: I79). Alors que, dans le pays de son père, il ne tomba pas une goutte de pluie pendant sept ans.

Le motif hagiographique de la vierge miraculeusement capable d'allaiter un nouveau-né semble contredire la logique narrative du récit, construit largement sur l'opposition entre fertilité et stérilité, liées entièrement à la présence ou à l'absence de l'héroïne. Cette logique est cependant partiellement sauvegardée par la motivation de la demande: non pas pour nourrir l'enfant de bouillie faite à partir de lait et de farine, mais pour se nourrir elle-même et, semble-t-il, apporter la prospérité aux gens qui l'accueillent. ${ }^{16}$ Ces versions détournent en quelque sorte le motif du pays frappé de stérilité auquel elle apporte l'abondance: les vaches qui donnent du lait à pleins seaux et le moulin qui produit des sacs de farine à partir d'un seul grain de blé. Sa demande ne serait pas justifiée par la nécessité d'alimenter un nouveau-né grâce à la nourriture donnée aux enfants par des femmes dans l'incapacité d'allaiter, puisque elle-même a obtenu de Dieu cette aptitude. Ces versions ainsi gauchies tentent de préserver une logique narrative, au prix, sans doute, de la portée signifiante du conte.

Qu'est-ce que ce récit raconte et cache de suffisamment important pour engendrer une expression narrative collective ? Il semble bien, on l'a dit, que ce conte soit de création relativement récente, à partir d'un schéma déjà là — « La Fille aux bras coupés » -, mais immédiatement autonome, ne serait-ce qu'en éliminant le plus souvent la mutilation et en remplaçant le ou les propres enfants de l'héroïne par un nouveau-né qu'elle n'a pas mis au monde mais qu'elle prend en charge complaisamment, comme un devoir auquel elle ne peut se dérober.

Ce conte a très certainement surgi au XIx ${ }^{\mathrm{e}}$ siècle dans la région du Nivernais et du Morvan, qui fournissait alors la plus grosse proportion de nourrices de toute la France. Elle est restée ${ }^{17} \mathrm{Au}$ cours du Xix ${ }^{\mathrm{e}}$ siècle, l'administration tenta d'orga-

[wet-nurse] de Dona Antonia). Je supposerais volontiers que la conteuse était en effet une de ces nourrices donnant son lait à des enfants étrangers.

I5 Elle a vécu dans la solitude d'un arbre creux — souvenir de « La fille aux mains coupées ». C'est l'enfant qui décide, au bout de sept ans, qu'ils ne peuvent plus rester là.

I6 Dans «Virginette ", elle a le vif désir de manger du pain, la nourriture constitutive de l'humanité, alors qu'elle avait vécu de racines (et de la grâce de Dieu) pendant sept ans.

I7 Direction des affaires sanitaires et sociales. Nous renvoyons à la belle étude d'Anne Cadoret (I995). 
niser et de contrôler ce vaste artisanat de la mise en nourrice des nouveau-nés, dont les causes étaient multiples. On différencie alors les nourrices « sur lieu », qui viennent s'installer dans la famille du nourrisson, et les nourrices «à emporter » qui gardent les enfants à domicile, le plus souvent à la campagne. Deux raisons peuvent expliquer que le Nivernais et le Morvan fournissent un gros contingent des deux catégories : la région est relativement proche de Paris et elle est très pauvre. Au XX $\mathrm{X}^{\mathrm{e}}$ siècle, après l'adoption sans risques de l'allaitement au biberon, cette activité ne concerne plus que l'accueil des enfants de l'Assistance publique et se justifie toujours par l'apport d'argent frais versé par l'administration, sans lequel de nombreuses familles n'auraient pu survivre, réduites à une économie quasi autarcique. Les exploitations agricoles, majoritairement de petite taille, laissaient un déficit de ressources que la maigre indemnité versée par l'Assistance publique permettait de pallier en partie. Anne Cadoret rapporte le témoignage d'un homme né en I9I6, arrivé à l'âge de quelques jours :

Je vins prendre le lait du dernier enfant, Jean, né deux mois après la mort de [son] père. C'est en effet cette détresse qui avait amené maman Claudine à se mettre à la disposition de l'Assistance publique, comme nourrice: donnant son lait, elle serait rétribuée davantage (I995: 80).

En tant que nourrices «sur lieu », les jeunes femmes issues de la région sont réputées dès le XIX ${ }^{e}$ siècle, même si elles n'en fournissent pas tout le contingent. Paris en draine un bon nombre, placé soit de gré à gré, soit par l'intermédiaire de «bureaux de nourrice » où les familles bourgeoises viennent choisir et engager une de ces femmes venant de la campagne, après qu'elle a confié son propre enfant aux soins d'une parente. Elles vivront un véritable exil, même si leurs conditions de vie matérielle sont plus favorables qu'au village, subissant les fortes contraintes qu'exercent sur elle les parents de l'enfant et le médecin de famille. On retrouve donc des éléments de l'histoire de Brigitte : à commencer par le départ forcé de son pays d'origine et la nécessité de nourrir un enfant qui n'est pas le sien.

L'étude des contes prend difficilement appui sur la réalité sociale et économique des groupes dans lesquels ils étaient transmis. Les tentatives d'interprétation directe, terme à terme, se révèlent souvent peu productives. Considérer le Petit Poucet (T 327B) comme le reflet de l'âpreté de la vie paysanne dans la société traditionnelle, qui mènerait les parents à l'abandon, ou à l'idée de l'abandon, de leurs enfants trop nombreux, est certes tentant mais ne permet pas de saisir l'acte créateur aboutissant au conte. Il existe cependant des liens étroits entre réalité et fiction, la première subit cependant une telle élaboration pour aboutir à la seconde qu'une lecture directe, terme à terme, est très aléatoire. Dans le cas du T 7I3, il faut noter en premier un important travail de sublimation : parfois jusqu'à transformer l'héroïne en sainte. Cette sainteté est à la hauteur de la souffrance que subissaient ces jeunes femmes obligées de laisser leur propre enfant pour nourrir un nouveau-né étranger, après avoir été choisie comme le serait une vache laitière, passant ensuite de nombreux mois, voire des années, loin de leur pays d'origine et de leurs parents et proches.

En revanche, elles apportaient la prospérité à leur retour au pays. Fanny Faÿ-Sallois rapporte que, dans les années I880, elles pouvaient recevoir entre soixante et quatre-vingts francs de salaire par mois. «Le montant est établi de gré à gré entre la nourrice et la famille, sur un marché où l'offre et la demande 
restent également abondantes, même si la première l'emporte légèrement sur la seconde » (I980: 200). Elle reçoit également des gratifications et des vêtements. À la même époque, une bonne à tout faire gagnait de vingt à trente francs. Elles se constituent ainsi, dit A. Cadoret, « un pécule suffisant pour que la pauvre masure morvandelle devienne une maison plus arrangée, "la maison de lait" » (I995: 87).

Le récit insiste sur la double prospérité qu'apporte l'héroïne là où elle se trouve. Exilée, elle arrive dans un pays stérile depuis des années, à laquelle elle redonne l'abondance en générant la nourriture nécessaire à l'enfant nouveau-né, alimentation de base, lait et farine..$^{8}$ Il est dit parfois qu'à son départ, elle laisse cette prospérité aux habitants du pays auxquels elle l'a procurée, promettant aux gens qui l'ont accueillie que leurs vaches donneront toujours du lait et qu'ils auront toujours du blé, comme dans la version intitulée "Philomène ». ${ }^{19}$ Peut-on y voir la figuration du don fait par la nourrice à la famille qui l'emploie: un enfant, non pas mis au monde par elle, mais auquel elle fait franchir cette période périlleuse, qui va de la naissance au sevrage? Et son retour au pays redonne à celui-ci la prospérité qu'il ne connaissait plus, ainsi que, dans la réalité vécue, un précieux bienêtre financier. La narration a sublimé de rudes réalités, sociales, économiques et

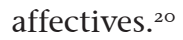

Nous avons posé l'hypothèse que ce conte merveilleux a été élaboré dans la région du Nivernais et du Morvan à une date relativement récente, ${ }^{2 \mathrm{r}}$ et qu'il a été reçu dans le sud-ouest de la France et jusqu'à l'île de Majorque grâce à un truchement féminin unique ou en très petit nombre. Mais le récit ne disposait pas localement d'un contexte social et économique aussi bien institutionnalisé que celui de son lieu d'origine. Pour se maintenir, il doit alors faire appel à un motif légendaire qui appartient à un fonds hagiographique connu, celui du lait qui afflue aux seins d'une personne, le plus souvent un homme pour mieux affirmer le caractère miraculeux de l'événement et qui témoigne éminemment de sa sainteté. Le motif n'appartient pas à l'univers du conte merveilleux, mais, dans cet exemple, il permet à un conte " déraciné » de se maintenir dans la transmission après sa migration. C'est au prix cependant d'un glissement vers la légende, au niveau de sa signification, même s'il conserve la forme organique du conte merveilleux. Le motif légendaire essentiel du point de vue narratif relève du miracle et non plus du merveilleux comme dans les versions nivernaises.

On pourrait objecter que la conjoncture est la même dans la réalité historique et dans les quatre versions méridionales, puisque, en effet, les jeunes nourrices nivernaises donnaient leur propre lait tout autant que les héroïnes qui, bien que vierges, voient le lait affluer à leurs seins. Pour ce qui est des versions nivernaises où les héroïnes n'ont pas de lait, j'y verrais, paradoxalement, un effet du passage à la narration grâce à la sublimation de la réalité. Dans cette réalité, les jeunes femmes privaient leurs propres nourrissons de leur lait pour le donner à des en-

I8 Si l'on s'en tient aux versions nivernaises.

I9 Dans la version d'Alcover, il est noté que les hôtes de l'héroïne trouvent du pain dans le panier à pain qui reste désormais toujours plein.

20 L'ouvrage de F. Faÿ-Sallois donne les détails de ce qu'elle appelle justement l' «industrie du lait » et décrit le folklore qui entoure le personnage de la nourrice, qui va de l'attendrissement au grotesque.

2I Fin du XVIII ${ }^{\mathrm{e}}$ ou début du xIX ${ }^{\mathrm{e}}$ siècle? 
fants étrangers. L'héroïne des contes nivernais, mise dans l'obligation de nourrir un enfant étranger, réussit à subvenir à ses besoins grâce à une nourriture d'origine cultivée (agriculture, élevage) qui est en quelque sorte générée par le besoin urgent qu'elle en a. ${ }^{22}$ Nous aurions, de ce côté, un appel à une ressource humaine, de l'autre un recours à une entité divine, laquelle dispense un miracle qui instrumentalise le corps de l'héroïne en lui octroyant du lait bien qu'elle soit vierge.

S'agit-il de la distinction, difficile à établir, entre mythique et miraculeux, entre conte merveilleux et légende ? Dans un article ancien, j'avais privilégié l'étude de l'élément mythique que constitue la différenciation faite entre deux sortes de mères, celle qui procrée l'enfant et celle qui le nourrit, en m'appuyant sur le rituel romain ancien des Matralia, où, dans le temple de Mater Matuta, les femmes et mères d'un premier mariage s'occupaient, non de leurs propres enfants, mais des enfants de leurs sœurs, ainsi que sur la figure de la divinité védique de l'Aurore, sœur de la Nuit, qui « prend livraison du fils de la Nuit et le soigne à son tour ». Georges Dumézil cite d'autres attestations laissant entendre cette double maternité (I973: 305-330). En des termes plus communs, dépourvus de toute connotation religieuse, le conte dit également que la maternité est une tâche double, la mise au monde et le nourrissage, que deux femmes peuvent assumer l'une après l'autre, sans qu'aucune d'elles ne soit plus mère que l'autre. Le conte merveilleux se préoccupe de problèmes concernant les liens familiaux, les relations de parenté perturbées, la prospérité locale, le destin des individus. Dans la communauté particulière que constituent le Nivernais et le Morvan, la question de l'allaitement des enfants, considéré le plus souvent ailleurs sur les seuls plans physiologique et affectif, perturbe suffisamment la collectivité pour qu'elle tente de la penser grâce aux moyens à sa disposition, à savoir le conte merveilleux qui expose les faits en termes littéraires, sublime la réalité pénible et lui invente des solutions satisfaisantes.

Le récit ne prend pas seulement en compte la question du dédoublement de la mère biologique et de la mère nourricière, il traite dans son épilogue de la remise en ordre de la parenté perturbée par ces événements. Sur les onze versions nivernaises, huit en parlent clairement. ${ }^{23}$ Ainsi dans « Brigitte » $\left(\mathrm{n}^{\circ} \mathrm{I}\right)$ :

Pour arriver, tout reverdissait; en arrivant, l'enfant dit : - Une pomme pour mon papa, le roi ; une autre, pour ma maman, la reine, qui m'a fait,

22 Il faudrait analyser plus avant la version intitulée "Santa Brigita » (Petit I974), où l'héroïne, ayant obtenu du Seigneur la possibilité d'allaiter l'enfant, s'exile d'elle-même avec le nourrisson car elle est stigmatisée comme fille-mère. Elle l'est également durant son exil, jusqu'à arriver près d'un moulin où se trouvent deux enfants dont les parents sont morts. C'est pour eux qu'elle génère successivement du bois de chauffage, du lait, de la farine; elle leur prépare également des lits, et par la suite recrée des jardins là où ceux-ci existaient autrefois. Ils vivent ensemble plusieurs années jusqu'à ce que le malheur qui affecte son pays d'origine soit attribué à l'absence de quelqu'un du village : le recensement indique qu'il s'agit de Brigitte. Le récit introduit une variante si cohérente avec la logique du récit qu'elle l'explicite un peu plus: le recours mythique aux ressources agricoles d'origine humaine. L'héroïne recrée un environnement de survie à l'usage de deux enfants orphelins, qu'elle peut se permettre de laisser lorsqu'ils sont devenus grands et autonomes.

23 Les trois autres sont très lacunaires, ne gardant que le motif de la prospérité et de la disette liées à la présence ou à l'absence de l'héroïne. 
pas nourri; une pour ma grand-mère qui m'a mis vers maman Brigitte qui m'a pas fait mais nourri. ${ }^{24}$

Dans « Brigitte » $\left(\mathrm{n}^{\circ} 4\right)$, lors du retour de la jeune fille que son père est venu chercher:

En passant sous un poirier, l'enfant dit: — Maman veux-tu que je cueille une? -_ On te gronderait. — Oh! j'en cueillerais seulement cinq : une pour maman qui m'a fait, mais pas nourri, une pour grand-père, une pour grand-mère, une pour toi qui m'a nourri, mais pas fait, une pour moi. Il les cueille. En arrivant, on mange et il fait cadeau des poires, en disant comme ci-dessus.

Trois des versions du sud-ouest connaissent ce motif de clôture: celles de Maugard, Arnaudin et Petit, cette dernière particulièrement touchante.

Cet homme emmena Brigitte et le petit qui était un peu grand. Quand il arriva dans son village, on fit une grande fête, c'était en été, ils mirent des tables tout le long de la route, on fit une fête terrible! A la fin du repas, Monsieur le Maire donna une pomme à ce petit; il lui dit: - Tiens, petit, va porter cette pomme à ta maman. Et le petit alla la porter à sa mère qui l'avait mis au monde. Mais on lui dit: - Tu te trompes, petit, ce n'est pas ta maman. - Oh, dit-il, si fêt, celle-ci est celle qui m'a mis au monde, et celle-là celle qui m'a nourri. Et alors tout le monde sut que ce n'était pas l'enfant de Brigitte.

Cet épisode de l'assignation de la parenté n'omet jamais la distinction entre la mère qui a « fait » l'enfant et celle qui l'a « nourri », entre la mère biologique et la mère nourricière, mais il étend parfois un peu plus le cercle familial. Dans les versions nivernaises, c'est toujours l'enfant qui désigne spontanément un nombre plus ou moins important de membres de la famille, famille dans laquelle il se réinsère lui-même en nommant les liens de parenté. Dans les versions du sudouest qui gardent le motif, il y est incité, soit par sa mère nourricière (Maugard), soit par le père de l'héroïne (Arnaudin) ou encore par le maire du village (Petit). En revanche, les versions nivernaises laissent penser que l'enfant reconstitue luimême le tissu familial lacéré par la trahison de la marâtre, cette autre sorte de mère. ${ }^{25}$

Trop dépendant d'un contexte social et économique qui a considérablement évolué en un siècle, le conte de Brigitte peinait déjà à l'époque de Millien à se constituer en récit accompli : en témoignent les versions nivernaises lacunaires, dont cependant le mode d'expression est très vigoureux. Pour s'implanter dans une région où la mise en nourrice existait comme simple pratique sociale et non comme ressource quasi « industrielle » selon le mot de F. Faÿ-Sallois, il lui fallait faire appel à d'autres moyens littéraires, ceux de la légende hagiographique. La modification du motif du nourrissage de l'enfant, obtenu grâce à une prière à

24 Dans cette version, les relations de parenté sont complexifiées par la conteuse.

25 A. Cadoret note la distinction faite clairement par la communauté entre « enfants de famille » et « enfants placés » et de façon sensible les subtiles distinctions langagières à l'intérieur des familles d'accueil: « C'est mon frère, mon frère, mais ça s'arrête là. C'est bien spécifique quand même entre dire mon frère et être mon frère » (I995: I49). Elle cite également le propos d'un ancien enfant assisté (on ne sait si c'est un homme ou une femme): «Ce n'est pas ma mère, mais c'est plus ma vraie mère que ma mère » (I989: II2). 
Dieu, si mince soit-elle apparemment, constitue un emprunt aux ressources littéraires de la légende, plus diffuses et donc mieux partagées, permettant de valider un conte inconnu jusque là dans cette région du sud-ouest de la France et de l'y maintenir, en peu d'exemplaires cependant. ${ }^{26}$ Ce glissement témoigne d'un usage libre des matériaux issus des différents genres de la littérature orale, libre mais cohérent, lorsque l'environnement social et économique ne permet plus de comprendre la nécessité impérieuse de penser et de sublimer les âpretés de certaines vies, féminines en l'occurrence.

\section{Références bibliographiques}

ARnAUdin, Félix (I994) : Contes populaires de la Grande-Lande. Parc national des Landes de Gascogne/Editions Confluences.

BELMONT, Nicole (I984): "Mythe et folklore. A propos du conte français T 7I3 ". Le Conte. Pourquoi ? Comment? Colloques internationaux du CNRS. Paris : CNRS Éditions, p. 379-392.

- (20I7): Petit-Poucet rêveur. La poésie des contes merveilleux. Paris: Librairie Corti.

CADORET, Anne (I989): Le devenir des enfants placés dans la Nièvre ou le jeu de la reproduction familiale. Rennes: Institut Régional du Travail Social de Bretagne, rapport de recherche.

— (I995) : Parenté plurielle. Anthropologie du placement familial. Nouvelles études anthropologiques. Paris: L'Harmattan.

Delarue, Paul (I956): Von Prinzen, Trollen und Herren Fro, Märchen der europäischen Völker, Jahresgabe 1956 der Gesellschaft zur Pflege des Märchengutes. Rheine i. Westf, p. 95-98.

- (I959) : «Le conte de "Brigitte, la maman qui m'a pas fait, mais m'a nourri" ». Fabula 2 (2): 254-264.

DuMÉZIL, Georges (I973): Mythe et épopée III. Histoires romaines. Paris: Gallimard. FAŸ-SALlOIS, Fanny (I980): Les Nourrices à Paris au XIX $X^{e}$ siècle. Paris: Payot.

GrimAlt, Josep A. (I986): «A Majorcan Version of AaTh 7I3 ». Fabula 27 (I): 46-53.

LAURENT, Donatien (I982) : «Brigitte, accoucheuse de la Vierge. Présentation d'un dossier ». Le Monde alpin et rhodanien $\mathrm{n}^{\circ} \mathrm{I}-4: 73-79$.

MAUGARD, Gaston (I952): «Le conte de Sainte-Germaine ». Folklore 67 (été I952): 23-28.

- (I955): Contes des Pyrénées. Contes merveilleux des Provinces de France. Paris: Erasme.

Petit, Joan Maria [I974]: «Contaires del Menerbés: Germana Cabrol ». Obradors (Centre d'Estudis Occitans de l'Universitat de Montpellièr). Novèla tièra 4: p. 48-5I.

26 La version la plus récemment collectée est celle de Petit: I974, contée en 1973 par Germana Cabrol, née en 1904 à Félines-Minervois (Hérault). 